\title{
Evidence Based Management of Aneurysmal Subarachnoid Haemorrhage: Audit of Current Practice
}

S White, M Heald, S Sunny, G Kakkar, A Augustine

University Hospitals of North Midlands W/HS

\section{Introduction}

Subarachnoid haemorrhage (SAH) accounts for $5 \%$ of all stroke in the UK, with overall mortality of $50 \%$. One-third of survivors are dependent for care and almost half will have cognitive impairment sufficient to affect their quality of life (1).

\section{Methodology}

-All patients undergoing endovascular coiling at University Hospital North Midlands from May to Oct 2014

-Retrospective data collection using a pro-forma developed from the AHA/ASA 2012 guidelines (2).

\section{Results}

- 21 patients: female $90 \%$, male $10 \%$

-Age: - Range 25 - 67 years

- Mean $54.5+/-11.3$ years

- Aneurysm - Single: $67 \%$, multiple $33 \%$

- Less than $7 \mathrm{~mm} 71 \%, \geq 7 \mathrm{~mm} \mathrm{29 \%}$

- $52 \%$ transferred from other hospitals

- $33 \%$ of patients had additional procedures: EVD, decompressive craniectomy

\section{Fig.1 Time to definitive treatment}

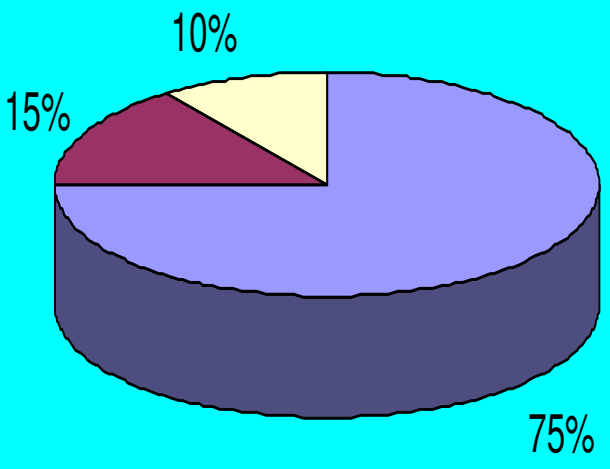

$\square$ within 24 hours $\quad \square 24-48$ hours $\quad \square$ more than 48 hrs

\section{Discussion}

Retrospective collection of data has limitations.

There is good access to interventional radiology services as evidenced by $90 \%$ of aneurysms being secured within 48 hours.

Blood pressure control was variable with clear episodes of hypertension in unsecured aneurysms.

Data on fluid balance was very sparse but perioperative haematocrit levels indicate that fluid administration could be increased.

Overall, peri-operative care was appropriate as evidenced from good outcomes at 6 months.

Potential areas for improvement:

- administration of nimodipine

- better peri-operative blood pressure control Future audit will be improved following introduction of a prospectively compiled data base.

\section{Conclusion:}

Good practice was observed at UHNM complying with the recommendations of the AHA/ASA for definitive treatment of aneurysm within $72 \mathrm{hrs}$. We are introducing a check-list style proforma with key interventions to be signed and dated in front of patient notes to improve and standardise care.

\section{References:}

1. Luoma A, Reddy U; Acute Management of aneurysmal subarachnoid haemorrhage; Continuing Education Anaesthesia Critcal Care Pain (2013) 13 (2): 52-58.

2. Connolly $E$ et al. Guidelines for the management of aneurysmal subarachnoid hemorrhage: a guideline for healthcare professionals from the American Heart Association/American Stroke Association. Stroke.2012;43:1711-1737. 